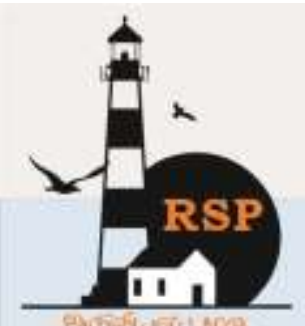

INTERNATIONAL RESEARCH JOURNAL ON ADVANCED SCIENCE HUB

- ISSN : $2582-4376$ Open Access

RSP SCIENCE HUB

[The Hub of Research ldeas]

Available online at www.rspsciencehub.com

\title{
Life Saving Ambulance Monitoring Using Image Processing
}

Ramya $P^{1}$, Priyadarshini ${ }^{2}$, Varun Kumar $K^{3}$, Vinitha Alamelu $G . K^{4}$

${ }^{1}$ Assistant Professor, Department of Electronics and Communication Engineering, SNS College of Engineering, Tamil Nadu, India.

2,3,4 UG Scholar, Department of Electronics and Communication Engineering, SNS College of Engineering, Tamil Nadu, India.

\begin{abstract}
Traffic clog and tidal stream the executives were perceived as serious issues in current urban regions, which have caused a lot of awkward for the rescue vehicle. The thought behind this plan is to execute an ITS which would control precisely the traffic lights dependent on the quantity of vehicle present in the way and furthermore dependent on the emergency vehicle signal. This plan is completely computerized, in this manner it finds the mishap spot, controls the traffic lights, assisting with arriving at the emergency clinic in time.
\end{abstract}

\section{Keywords: Traffic lights, Emergency clinic, Vehicle signal.}

\section{Introduction}

Vehicular traffic control at street intersections has consistently been a matter of worry for organizations in numerous advanced urban communities around the globe. It is confronting horrendous street clog issues in its urban communities. Framework development is delayed when contrasted with the development in various vehicles, because of space and cost. A few endeavours have been made to structure productive computerized frameworks to settle this issue. The greater part of the present-day frameworks uses pre-decided timing circuits to work traffic signals which are not very effective in light of the fact that they don't work as indicated by the current volume of traffic at the intersection. It is frequently found in todays mechanized. traffic control frameworks that ambulances are getting made up for lost time by a red traffic sign and burning through significant time.[1-4] Blockage is frequently converted into lost time, missed opportunities, lost specialist efficiency, conveyance delay and, as a rule expanded cost.
Time is the basic factor deciding whether a crisis activity will be effective. Its an incredible test for crisis vehicles to find a workable pace scene in a safe way rapidly, particularly when traffic turns out to be progressively overwhelming and traffic design develops increasingly complex in current cities. Emergency vehicle stalls out alongside a long stack of different vehicles in front that is halted because of red sign, Brilliant guide is intended to control the traffic flags along the way of the rescue vehicle, when an emergency vehicle moves toward an convergence the traffic light is changed to green and when the emergency vehicle passes the convergence the control is reestablished to the correct sign counterbalance esteem once more. Presenting an IoT-based plan for the framework, [5-9] it very well may be for all intents and purposes be controlled from anyplace. Makes it all around controllable and expands the responsive viability. It tends to be utilized in circumstances like traffic blockage, crisis the executives, VIP escort etc. Thus, the framework builds the chance of sparing a real existence. 


\section{Literature Survey}

A composing diagram is an assortment of substance that plans to study the essential motivations behind current data counting substantive disclosures similarly as speculative and methodological responsibilities to a particular point. Composing reviews are assistant sources and, for instance, don't report any new or one of a kind preliminary work. Routinely associated with insightful arranged composition, for model, these, composing generally speaking goes before an investigation recommendation and result fragment. Its conclusive objective is to convey the per client completely educated in regards to the stream composing on atopic and structures the purpose behind another goal, for instance, future research that may be required in the domain. An inside and out sorted out composing review is depicted by a real movement of contemplations,[10-12] rhythmic movement and pertinent references with dependable, fitting style; suitable usage of wording and a fair-minded and intensive viewpoint on the past research on the point. Developed an improvement prepared instrument for crisis vehicle journey by (A2FAP) for Indian circumstances. This explore basically uses the present headways close by the thought called web of things (IoT). The structure used is server-client building. The client is a customer using an android application. Have executed VANET-based emergency vehicle advised system. They have arranged an emergency vehicle advised system that uses between vehicle correspondence and besides encompasses side of the street establishment like traffic lights. At the present time, vehicles are forewarned of a pushing toward emergency vehicle and moreover get clever dirty course information. Considering this information, an advantageous and legitimate reaction of various drivers is possible.

Proposed the microcontroller-based RFID structure that is used to change the traffic lights after arriving in busy time gridlock light crossing point. The system makes an android application that interfaces both the crisis vehicle and the traffic signal station using cloud compose. This structure uses Radio repeat conspicuous evidence advancement to execute the watchful traffic signal control. The basic idea behind the proposed system is, if the Ambulance finishes in travel due to a traffic signal, RFID presented at the traffic signal tracks the RFID named crisis vehicle and sends the data to the cloud. After the insistence for the customer through the versatile application, the explicit sign is made Green for a long time and after the salvage vehicle travels by, it recoups it's one of a kind movement of the gathering of hailing, if this arrangement is totally robotized, it finds the crisis vehicle spot, controls the traffic lights. This system controls the traffic lights and saves the time during emergency periods.

\section{Existing System}

Traffic blockage is a basic issue in a nation like India which is exceptionally populated. Metropolitan urban communities are bonded to the unforgiving truth of traffic blockage. A normal human goes through as long as 4 hours in a day in rush hour gridlock. Right now, a crisis vehicle such a rescue vehicle needs to arrive at a goal then there is a high measure of time that is spent in making room. Each second spent on the freedom cost the life of the patient. Despite the fact that there are numerous ideas related to traffic control and crisis vehicle freedom, none of these ideas were actualized in the nation. From the past decades, the board of traffic has been one of the greatest issues of modernization. Analysts have followed far to defeat the traffic emergencies. Directly from the earliest starting point of Manual Traffic Control in which labour was required to control the traffic. Contingent upon nations and states the traffic approaches are designated to various regions to control traffic.

These men convey sign board, sign light and whistle to control the traffic. They are told to wear explicit regalia in request to be effortlessly recognized by the drivers. After this came the conventional Vehicle Actuated Control System in which, lights are stacked with the steady numerical incentive in the structure of clocks. The lights are naturally jumping $\mathrm{ON}$ and $\mathrm{OFF}$ contingent upon clock esteem changes. The primary burden is that the calculation for this control framework doesn't change the green sign regardless of whether the traffic has just gone until the counter is finished, while not considering the number of vehicles holding up at red.There were numerous activities distributed in the ongoing years identified with crisis vehicle leeway and they all meet to a similar thought i.e Emergency vehicle leeway utilizing RFID tag. Right now, every vehicle is furnished with a uncommonly prepared RFID (Radio Frequency Identification) tag furthermore, each traffic signal is furnished with a RFID per user. This framework proposes that when an emergency 


\section{www.rspsciencehub.com}

vehicle is approaching a traffic signal point, the RFID per user peruses the RFID label installed in the vehicle and turn the sign green until the rescue vehicle passes.

The weakness with this framework is that there is no focal observing procedure to check the status of the vehicle if there should arise an occurrence of any disappointment, there is a need to actualize a RFID tag what's more, RFID per user to each traffic signal point which isn't cost effective. What extremely motivative us to build up this undertaking was that there were many existing ideas identified with crisis vehicle the board however none of these were actualized in the nation. With the Internet of Things spinning around with expanded correspondence between machine to machine; its based on distributed computing and systems of information gathering sensors; its portable, virtual, and quick association; and its said that it will make everything from streetlights to seaports keen. This cleared path to the idea of including an IoT based answer for the traffic the executive's framework, the Brilliant Aid. This IoT based arrangement can be for all intents and purposes controlled by anyplace and builds the responsive adequacy.

The existing system that are already proposed are given below:

- $\quad$ Time based Traffic Control.

- Fails to track the collision and pre-damage status. Time delay (first aid).

\section{Proposed Work}

Traffic signal is turned dependent on the quantity of vehicle present in the street. Another plan for consequently controlling the traffic flags and accomplishing the previously mentioned task so the rescue vehicle would have the option to cross all the traffic intersections without pausing. Remote advances utilized for data moving. The structure and usage of Golden Aid is straightforwardly directed for traffic the executives with the goal that crisis vehicle on street gets clear approach to arrive at their goal in less time what's more, with no human obstruction. This can be accomplished by naturally changing the sign ahead green when the ambo- spear is approaching it.

This framework attempts to give correspondence among emergency vehicle and
Volume 02 Issue 03 March 2020

different gadgets, for example, traffic signals. The framework comprises of a concentrated traffic the board framework that screens the situation of ambulances in genuine time and deals with the traffic signals accordingly. The ambo- spear will continually send its GPS directions to the focal server. The focal server will store the areas of all the traffic focuses at first in the framework itself. Once an emergency vehicle approaches a sign which is red at that point, the focal server will trigger a computerized control to change that signal ahead into green. This system would make the way ahead of ambulances in rush hour gridlock signal intersections where the fundamental issue of blockage consistently exists. There are four modules in the framework:

- Automatic signal control without manpower.

- Accurate level of vehicles density was monitoring by using of camera section.

- Using Open CV Number of vehicles was counted at each side of lanes and then based on this count's vehicle density was predicted.

Using of Arduino Microcontroller vehicle density was collected from the Open CV, PC and the Traffic signal was changed corresponding to this information. The Block diagram of Proposed system shown in fig 1

\section{Block Diagram}

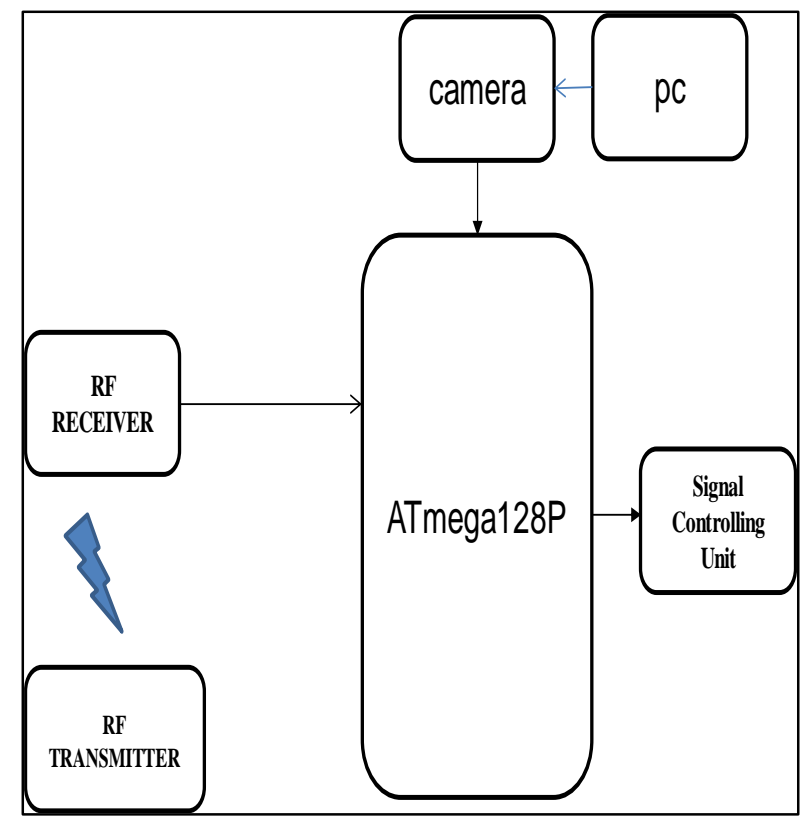

Figure.1. Block diagram of Proposed system 
modification R2) balanced as a USB-to-dynamic converter.

\section{Hardware Requirements}

6.1 ATMEGA 128p (UNO): The Arduino Uno board has 14 moved I/O pins (six fit for PWM yield), 6 direct I/O sticks, and is programmable with the Arduino IDE (Integrated Development Environment), by procedures for a canny B USB interface. The Atmega 128p(UNO) shown fig.2

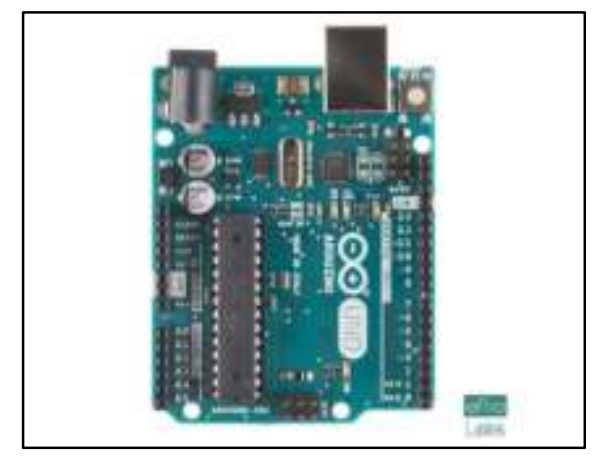

Figure.2. Atmega 128p(UNO)

The Arduino Uno board has 14 moved I/O pins (six fit for PWM yield), 6 direct I/O sticks, and is programmable with the Arduino IDE (Integrated Development Environment), by procedures for a canny B USB interface. It might be filled by the USB partner or by an outside 9-volt battery, at any rate it sees voltages some spot in the degree of 7 and 20 volts. It is moreover similar to the Arduino Nano and Leonardo. The gear reference course of action is drifted under a Creative Commons Attribution Share-Alike 2.5 license and is open on the Arduino site. Plan and creation documents for unequivocal sorts of the gear are in like manner open.

"Uno proposes "one" in Italian and was picked to check the essential appearance of Arduino Software. The Uno board is the first in an improvement of USB-based Arduino sheets; it and change 1.0 of the Arduino IDE were the reference sorts of Arduino, which have now progressed to additional pivotal releases. The ATmega328 on the board comes prearranged with a bootloader that grants moving new code to it without the utilization of an external hardware engineer.

While the Uno passes on using the first STK500 show up, it contrasts from each and every going before board in that it doesn't use the FTDI USBto-dynamic driver chip. Or on the other hand maybe, it uses the Atmega16U2 (Atmega8U2 up to

\subsection{LED:}

LED means "light emitting diode." A diode is an electrical part with two terminals which lead the power just one way. With an electrical flow, the diode radiates a brilliant light around the little bulb. It is a $\mathrm{p}-\mathrm{n}$ intersection diode that radiates light when enacted. At the point when a reasonable voltage is applied to the leads, electrons can recombine with electron openings inside the gadget, discharging vitality as photons shown in fig 3 .

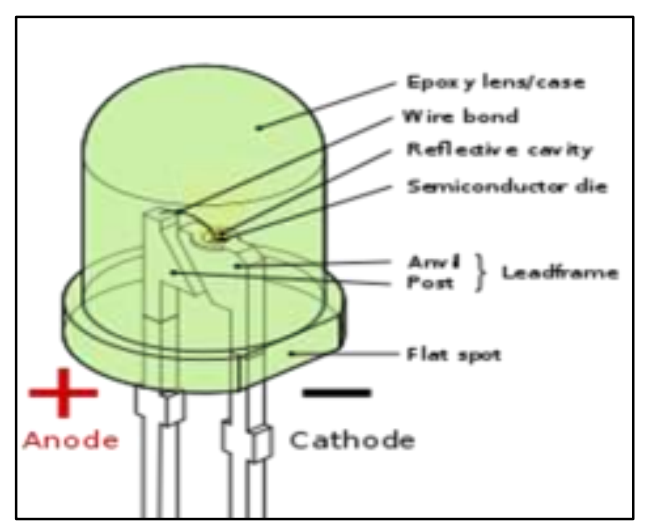

Figure.3. LED

LED means "light emitting diode." A diode is an electrical part with two terminals which lead the power just one way. With an electrical flow, the diode radiates a brilliant light around the little bulb. It is a $\mathrm{p}-\mathrm{n}$ intersection diode that radiates light when enacted. At the point when a reasonable voltage is applied to the leads, electrons can recombine with electron openings inside the gadget, discharging vitality as photons.

\subsection{Camera:}

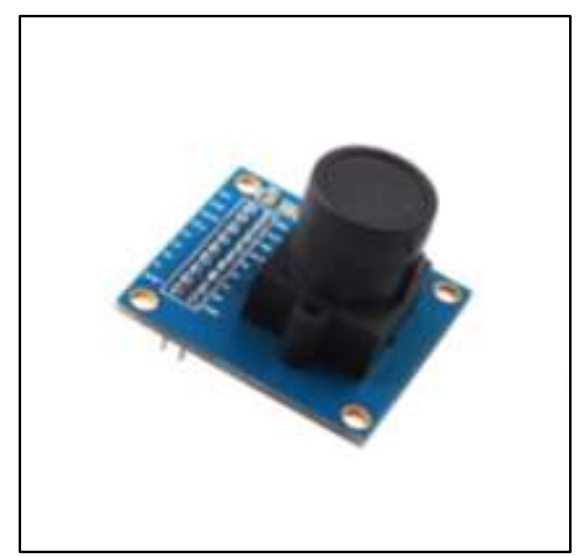

Figure.4.Camera 
A camera is an optical instrument used to record pictures. At their generally fundamental, cameras are fixed boxes (the camera body) with a little opening (the gap) that let light in to catch a picture on a light-touchy surface (typically photographic film or an advanced sensor). Cameras have different instruments to control how the light falls onto the light-touchy surface. Focal points centre the light entering the camera, the size of the opening can be enlarged or limited to give pretty much light access to the camera, and a screen instrument decides the measure of time the photograph touchy surface is presented to the light shown in fig 4.

The still picture camera is the primary instrument in the craft of photography and caught pictures might be recreated later as a piece of the procedure of photography, advanced imaging, photographic printing. The comparative masterful fields in the moving picture camera area are film, videography, and cinematography. The cuttingedge photographic camera developed from the camera obscure. The working of the camera is fundamentally the same as the working of the human eye. The main lasting photo was made in 1825 by Joseph Nicephore Niepce.

\subsection{Switch:}

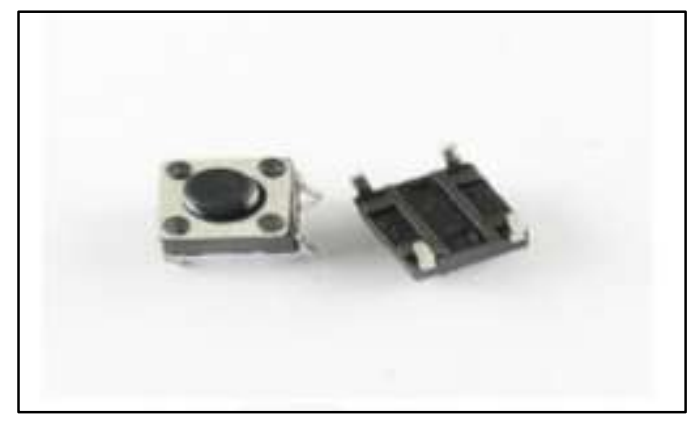

Figure.5. Switch

The most widely recognized kind of switch is an electromechanical gadget comprising of at least one lots of portable electrical contacts associated with outer circuits. At the point when a couple of contacts is contacting current can go between them, while when the contacts are isolated no current can stream.

Switches are made in a wide range of arrangements; shown in fig 5. They may have different arrangements of contacts constrained by a similar handle or actuator, and the contacts may work at the same time, consecutively, or then again. A switch might be worked physically, for instance, a light switch or a console button, or may work as a detecting component to detect the situation of a machine part, fluid level, weight, or temperature, for example, an indoor regulator. Many specific structures exist, for example, the flip switch, turning switch, mercury switch, press button switch, turning around switch, transfer, and electrical switch. A typical use is control of lighting, where different switches might be wired into one circuit to permit helpful control of light apparatuses. Switches in powerful circuits must have exceptional development to forestall dangerous arcing when they are opened.

\section{Software Requirements 7.1 Python:}

The least complex type of inserting Python is the utilization of the elevated level interface. This interface is proposed to execute a Python content without expecting to connect with the application legitimately. This can for instance be utilized to play out some procedure on a document.

Python is a deciphered, elevated level, broadly useful programming language. Made by Guido van Rossum and first discharged in 1991, Python's structure theory underlines code coherence with its remarkable utilization of noteworthy whitespace. Its language develops and object-arranged methodology mean to assist software engineers with composing clear, legitimate code for little and enormous scope ventures. Python is powerfully composed and trash gathered.

It bolsters different programming ideal models, including procedural, object-arranged, and utilitarian programming.

Python is frequently depicted as a battery included; language because of its exhaustive standard library. Python was considered in the late 1980 s as a successor to the ABC language. Python 2.0, discharged in 2000, presented highlights like rundown understandings and a trash assortment framework fit for gathering reference cycles. Python 3.0, discharged in 2008, was a significant correction of the language that isn't totally in reverse good, and a lot of Python 2 code doesn't run unmodified on Python 3.

The Python 2 language, for example Python 2.7.x, was formally stopped on 1 January 2020 (first got ready for 2015) after which security patches and different upgrades won't be discharged for it. With Python 2's finish of-life, just Python 3.5.x and later are bolstered. Python translators are 


\section{www.rspsciencehub.com}

accessible for some working frameworks. A worldwide network of software engineers creates and looks after C-Python, an open source reference usage. A non-benefit association, the Python Software Foundation, oversees and coordinates assets for Python and C-Python improvement.

\subsection{Embedded C:}

Embedded $\mathrm{C}$ is a lot of language augmentations for the $\mathrm{C}$ programming language by the $\mathrm{C}$ Standards Committee to address shared characteristic issues that exist between $\mathrm{C}$ expansions for various installed frameworks.

Embedded $\mathrm{C}$ programming normally requires nonstandard augmentations to the $\mathrm{C}$ language so as to help improved chip highlights, for example, fixed-point number juggling, numerous particular memory banks, and fundamental I/O activities. In 2008, the C Standards Committee stretched out the $\mathrm{C}$ language to address such capacities by giving a typical standard to all usage to hold fast to. It incorporates various highlights not accessible in typical C, for example, fixed-point number juggling, named address spaces and fundamental I/O equipment tending to. Inserted $\mathrm{C}$ utilizes the greater part of the language structure and semantics of standard C, e.g., primary () work, variable definition, datatype announcement, contingent articulations (if, switch case), circles (while, for), capacities, clusters and strings, structures and association, bit tasks, macros, and so forth.

\subsection{Open Computer Vision:}

OpenCV (Open Source Computer Vision Library) is an open source $\mathrm{PC}$ vision and $\mathrm{AI}$ programming library. OpenCV was worked to give a typical foundation to PC vision applications and to quicken the utilization of machine observation in the business items. Being a BSD-authorized item, OpenCV makes it simple for organizations to use and alter the code.

A full-highlighted CUDA and OpenCL interfaces are as a rule effectively grew at the present time. There are more than 500 calculations and around 10-fold the number of capacities that make or bolster those calculations. OpenCV is composed locally in $\mathrm{C}++$ and has a templated interface that works consistently with STL compartments.'

\section{Result \& Future Work}

The right now created framework is completely working what not the fundamental functionalities are met effectively relying on the prerequisites. In any case, so as to be utilized as an item in the certifiable certain adjustments must be done since it has to work and register progressively. Those progressions include: The result circuit shown in fig 6 .

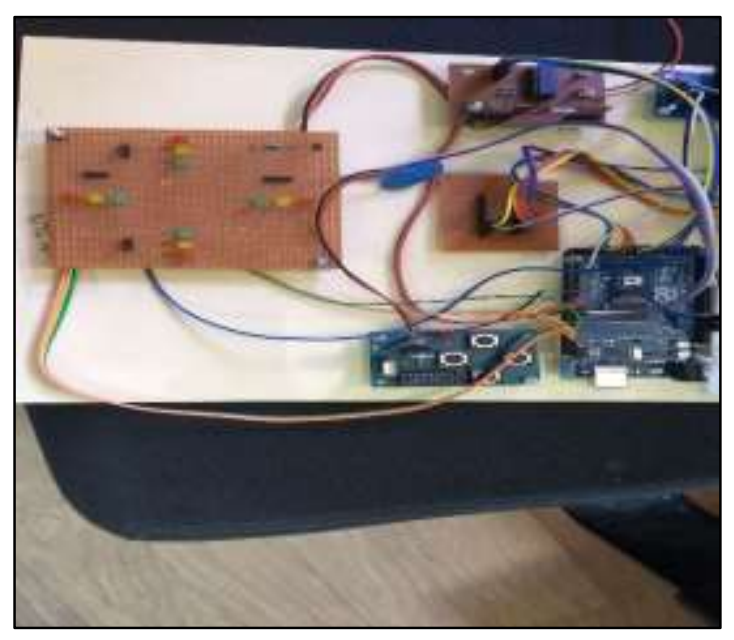

Figure.6. Result

- The focal server can be redesigned from Thing speak to a particular devoted server for this reason.

- Multi-threading must be accomplished all the more viably for expanding the framework responsiveness

- Additional security layers must be introduced. There can't be any odds of a security escape clause in a genuine time traffic framework like this.

- The circumstance of different ambulances moving toward a common traffic signal point must be considered with additional number of integral factors with the goal that the circumstance could be taken care of in the best and most effortless manner.

- The framework ought to have the option to fuse other emergency vehicles like police, fire and salvage and so forth with distinctive need levels.

\section{Acknowledgement}

The authors would like to thank Dr. S. Charles, Principal- SNSCE for supporting the experimental measurement and our sincere thanks to SNS College of Engineering for providing an equipped environment. 


\section{References}

[1] Sk. Riyazhussain, C.R.S. Lokesh, P.Vamsikrishna, Goli Rohan, et.al., "Raspberry Pi Controlled Traffic Density Monitoring System", IEEE WiSPNET 2016 conference

[2] Ossama Younis, Nader Moayeri, et.al., "Cyber-Physical Systems: A Framework for Dynamic Traffic Light Control at Road Intersections" IEEE Wireless Communications and Networking Conference (WCNC 2016)-Track 4Services, Applications, and Business

[3] M. Ashwin Kumaar, G. Akshay Kumar, S.M. Shyni, et.al., "Advanced Traffic Light Control System Using Barrier Gate and GSM", 2016 International Conference on Computation of Power, Energy Information and Communication (ICCPEIC)

[4] Jinyang Li, Yuanrui Zhang, Yixiang Chen, et.al., "A Self Adaptive Traffic Light Control System Based on Speed of Vehicles", 2016 IEEE International Conference on Software Quality, Reliability and Security Companion

[5] Syed Misbahuddin, Junaid Ahmed Zubairi, Abdulrahman Saggaf, Jihad Basuni, Sulaiman A-Wadany and Ahmed Al-Sofi, et.al.," IoT Based Dynamic Road Traffic Management for Smart Cities" 2012 45th Hawaii International Conference on System Sciences pp 2289-2297.

[6] Shiv. H. Sutar, Rohan Koul, Rajani Suryavanshi, et.al., "Integration of Smart Phone and IOT for development of Smart Public Transportation System",2016 International Conference on Internet of Things and Applications (IOTA) Maharashtra Institute of Technology, Pune, India 22 Jan - 24 Jan, 2016

[7] Hon Fong Chon, et.al., "Development of IoT Device for Traffic Management System", Lee Kong Chian Faculty of Engineering

[8] Chen Hui, Wang Xianghui, Zhang Xiqiang, Zhang Shaol et.al. ,"The evaluation of Chinese urban traffic management System Application Based intelligent traffic control technology", 2014 7thInternational Conference on Intelligent Computation Technology and Automation 978147996636-3/14 \$31.00 (C) 2014 IEEEDOI 10.1109/ICICTA.2014.191791

[9] Christoph Stoegerer, Thomas Novak, Wolfgang Kastner, Lukas Krammer, et.al., "Procedure based availability SL As for Traffic Management Systems", published in IEEE ETFA 2011Abstract

[10] Saed Tarapiah, Shadi Atalla, Basim Alsayid, et.al., "Smart On-Board Transportation Management System GeoCasting Featured" published in IEEE 2014 Road Transportation system.

[11] Mikulski Jerzy, et.al., "Katowice as a city with an Intelligent Traffic Management System", published in 20141EEE

[12] S. P. Kumar and O. Pandithurai, "Sixth sense technology," in Proc. of 2013 Int. Conf. on Information Communication and Embedded Systems (ICICES), pp. 947-953, February 21-22, 2013. 